\title{
Deubiquitinase Inhibitor Auranofin Attenuated Cardiac Hypertrophy by Blocking NF-kB Activation
}

\author{
Ming Hu Zhenhui Zhang $^{\mathrm{b}} \quad$ Bin Liu ${ }^{\mathrm{a}} \quad$ Shuangwei Zhang ${ }^{\mathrm{c}}$ Renjie Chai ${ }^{\mathrm{a}}$ \\ Xiaohua Chen $^{\mathrm{b}}$ Tianyu Kong ${ }^{\mathrm{b}}$ Fangcheng Zhang ${ }^{\mathrm{a}}$ Jingzhi Zhang ${ }^{\mathrm{c}}$ Shiming Liu ${ }^{\mathrm{a}}$ \\ Ningning Liu $^{\mathrm{a}}$ \\ aGuangzhou Institute of Cardiovascular Disease, the Second Affiliated Hospital, Guangzhou Medical \\ University, Guangzhou, Guangdong, 'Department of Critical Care Medicine, the Second Affiliated \\ Hospital, Guangzhou Medical University, Guangzhou, Guangdong, 'Department of Traditional Chinese \\ Medicine, the Second Affiliated Hospital of Guangzhou Medical University, Guangzhou, Guangdong, \\ China
}

\section{Key Words}

Auranofin $\cdot$ Cardiac hypertrophy $\cdot$ Deubiquitinase $\cdot$ Proteasome $\cdot$ NF-kB

\begin{abstract}
Background/Aims: Cardiac hypertrophy is a major outcome and compensatory response of the cardiovascular system to hemodynamic and additional stress responses that ultimately lead to heart failure. Auranofin (Aur) has been used for treating rheumatic arthritis for several decades. Aur is a $19 \mathrm{~S}$ proteasome-associated deubiquitinase inhibitor, and inhibition of the proteasome is speculated to reverse cardiac hypertrophy. However, the role of the deubiquitinases, especially $19 \mathrm{~S}$ proteasome-associated deubiquitinases, in the regulation of cardiac remodeling remains poorly understood. The present study investigated the role of Aur in cardiac hypertrophy both in vitro and in vivo. Methods: Male Sprague-Dawley rats underwent abdominal aortic constriction to induce left ventricular hypertrophy. The neonatal rat primary myocardial cell hypertrophy model was induced by Ang II. Echocardiography, hematoxylin-eosin staining, Masson's trichrome staining, immunochemistry, western blot analysis, a cell viability assay, and enzyme-linked immunosorbent assay were performed. Results: Aur significantly reduced the abdominal aortic constriction that led to left ventricular hypertrophy, reduced heart cavity expansion, and functional disorder, and thereby reduced fetal gene expression and attenuated cardiac fibrosis. Furthermore, Aur caused marked accumulation of ubiquitinated proteins and $\mathrm{I} \kappa \mathrm{B} \alpha$, as well as inactivation of NF- $\kappa \mathrm{B}$. This phenomenon was confirmed in the neonatal rat primary myocardial cell hypertrophy model. Conclusions: The present study indicated that Aur blocks the development of left ventricular hypertrophy induced by abdominal aortic constriction. This phenomenon might be attributed to inhibition of the 195 proteasome-associated deubiquitinase that can lead to aggregation
\end{abstract}

M. Hu, Z-h. Zhang and B. Liu contributed equally to this study.

Ningning Liu

and Shiming Liu
Guangzhou Institute of Cardiovascular Disease, the Second Affiliated Hospital, Guangzhou Medical University, 250 Changgangdong Road, Guangzhou, Guangdong (China); E-Mail Inn425@163.com, liushiming@gzhmu.edu.cn 


\section{Cellular Physiology Cell Physiol Biochem 2018;45:2421-2430 \\ \begin{tabular}{c|c|c} 
DOI: 10.1159/000488230 & and Biochemistry Published online: March 19, 2018 & $\begin{array}{l}\text { O 2018 The Author(s). Published by S. Karger AG, Basel } \\
\text { www.karger.com/cpb }\end{array}$
\end{tabular} \\ Hu et al.: Auranofin Attenuated Cardiac Hypertrophy by Blocking NF-KB Activation}

of IкB $\alpha$ and inactivation of the NF-кB pathway. Thus, Aur could be a potential anti-cardiac hypertrophy agent.

\section{Introduction}

Cardiac hypertrophy is a common pathological outcome of hypertension, myocardial infarction, coronary heart disease, and other cardiovascular diseases. Sustained cardiac hypertrophy eventually leads to heart failure. Several therapeutic approaches have been used extensively in the treatment of cardiac hypertrophy and general cardiac failure. Agents that block the adrenergic receptors include inhibitors of angiotensin converting enzyme (ACEIs) and $\mathrm{Ca}^{2+}$ channel antagonists [1]. Despite recent advances in drug therapy for cardiac hypertrophy and heart failure, these conditions have an extremely poor survival rate with an annual mortality rate of $29.6 \%$ [2]. Therefore, novel therapeutic agents that are effective in preventing or reversing the development and progression of cardiac hypertrophy would be indispensable.

In eukaryotic systems, the ubiquitin-proteasome system (UPS) is the primary protein degradation system. This pathway not only removes misfolded proteins but also performs crucial functions by regulating other systems including cellular growth and metabolic regulation of the cell cycle, DNA repair, and the activation of immunity $[3,4]$. Accumulating evidence shows that the development of cardiac hypertrophy is modulated by the UPS. Other studies have found enhanced proteasome expression and activity in cardiac muscle following pressure overload $[5,6]$. Currently, an effective therapeutic option for cardiac hypertrophy is speculated to be the functional modulation of proteasomes and regulation of their components.

Lee et al. found that treatment with proteasome inhibitors, namely, MG132 and bortezomib, for $24 \mathrm{~h}$ regressed cardiac hypertrophy induced by cholesterol in H9c2 cells [7]. In the pressure overload-induced cardiac hypertrophic rat model, proteasome inhibition clearly attenuated cardiac fibrosis and heart failure [8]. Our previous study also showed that the proteasome inhibitor Chinese herbal monomer compound known as gambogic acid significantly improved right heart and left ventricular hypertrophy in rats subjected to hypoxia and pressure overload $[9,10]$. The $19 \mathrm{~S}$ proteasome contains three deubiquitinases (DUBs): RPN11, UCH37, and USP14. These enzymes are responsible for degradation of the control protein [11]. Inhibition of 19S proteasome DUB activity can cause high levels of ubiquitin protein aggregation and the regulation of protein degradation. Moreover, 19S proteasome inhibition provides greater specificity and selectivity than $20 \mathrm{~S}$ proteasome inhibition [12]. Although it has been previously shown that $20 \mathrm{~S}$ proteasome inhibitors can suppress or rescue cardiac hypertrophy, it is not yet clear whether $19 \mathrm{~S}$ proteasomeassociated DUB inhibitors are effective in treating cardiac hypertrophy.

The gold (I)-containing agent, auranofin (Aur), has been used as a potent and effective compound in the clinical treatment of rheumatoid arthritis for more than three decades. In addition, Aur exhibits multiple activities that could cure other diseases. In addition to the anti-inflammatory activities, recent studies have shown that Aur exhibits potent antitumor effects against several carcinomas $[13,14]$. Previously, we showed that Aur is a potent and selective inhibitor of USP14 and UCHL5, two critical DUBs that are associated with the $19 S$ proteasome $[15,16]$. As observed with the 20 S proteasome inhibitor, Aur induces the accumulation of ubiquitinated proteins, blocks the breakdown of IкB $\alpha$, and subsequently inactivates functional NF- $\kappa B$ nuclear signaling [16]. In addition, the inactivation of NF- $\kappa B$ signal transduction suppresses cardiac remodeling induced by pressure overload $[17,18]$. Thus, the present study speculated that Aur is capable of suppressing pressure overloadinduced cardiac hypertrophy by a mechanism that is partially dependent on inhibiting proteasome activity and the NF- $\kappa \mathrm{B}$ signaling pathway. 


\section{Cellular Physiology Cell Physiol Biochem 2018;45:2421-2430

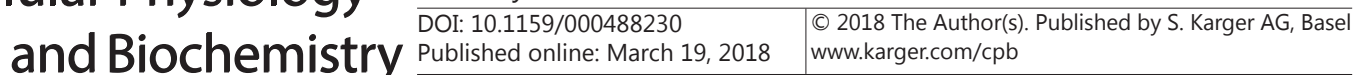

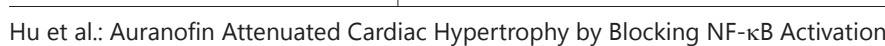

\section{Materials and Methods}

\section{Reagents}

Aur was purchased from Enzo Life Sciences International, Inc. (Plymouth Meeting, PA, USA). The following antibodies were used: anti- $\beta$-MHC (clone B-5), anti-ub (clone P4D1), anti-collagen type- 1 (Santa Cruz, CA, USA), anti-K48-linkage-specific polyubiquitin (clone D9D5), anti-IкB $\alpha$ (clone L35A5; Cell Signaling Technology, Beverly, MA, USA), and anti-GAPDH (Bioworld Technology, Inc.) Enhanced chemiluminescence (ECL) reagent was used (Santa Cruz) to develop the western blot membranes.

\section{Animals}

All animals were raised and treated in accordance with the guidelines by Guangdong Animal Center for the ethical treatment of animals. The experimental protocols were approved by the Institutional Animal Care and Use Committee of Guangzhou Medical University (Guangzhou, China). Male Sprague-Dawley rats (aged 6 weeks) were obtained from the Guangdong Laboratory Animal Monitoring Institute. All animals had free access to standard laboratory diet and water.

\section{Induction of left ventricular hypertrophy via abdominal aorta constriction (AAC)}

The animal models were created as described previously [19]. Four groups of rats were derived by randomly allocating 8 animals into each of the following groups: 1) vehicle-treated sham group (sham group); 2) Aur-treated sham group (sham+Aur group); 3) vehicle-treated AAC group (AAC group); 4) Aurtreated AAC group (AAC+Aur group). Briefly, the animals were anesthetized via intraperitoneal injection of $2 \%$ sodium pentobarbital $(2 \mathrm{~mL} / \mathrm{kg}$ ). Next, a midline abdominal incision exposed the abdominal aorta, and silk sutures (5-0) were used to ligate the aorta using a 22-gauge blunt-ended needle. The needle was rapidly retracted to create a defined constriction. For the sham group, an identical surgical procedure was applied in the absence of aortic constriction. Seven days post-surgery, the sham+Aur and AAC+Aur groups were given intraperitoneal injections of $3 \mathrm{mg} / \mathrm{kg}$ Aur (Enzo, USA) daily for 8 weeks. The rats of the sham and AAC groups received an equivalent volume of the vehicle to act as controls.

\section{Purification of neonatal rat cardiomyocytes (CMs)}

Neonatal rat CMs were isolated as described previously [20]. Briefly, the left ventricles were extracted from 1-2-day-old rats, sliced into small pieces, and digested with collagenase (Worthington Biologicals, USA) at $37^{\circ} \mathrm{C}$. Cells were pelleted and resuspended in Dulbecco's modified Eagle's medium (DMEM) containing $10 \%$ fetal bovine serum, followed by differential pre-plating to enrich the CMs, which were seeded in DMEM containing $0.1 \mathrm{mM}$ bromodeoxyuridine. The culture media were replaced with serum-free media after $48 \mathrm{~h}$ incubation and CMs were cultured for an additional $24 \mathrm{~h}$ prior to treatment.

\section{Cell viability assay}

MTS assay was performed to assess cell viability as described previously [21-23]. Briefly, the CMs were seeded in a 96-well plate. After treatment, MTS solution was added into each well and the cells were incubated for an additional $2 \mathrm{~h}$. The absorbance was read on a microtiter plate at a wavelength of $490 \mathrm{~nm}$.

\section{Histological examination of cardiac tissue}

Rats were killed by cervical dislocation at designated times post-Aur therapy. Formalin-fixed cardiac tissue sections were embedded in paraffin and cut into 5- $\mu \mathrm{m}$ thick slices. These sections were processed for staining by hematoxylin-eosin, Masson's trichrome, and immunohistochemistry protocols.

\section{Western blot analysis}

Briefly, equivalent amounts of total protein extracted from heart tissues or CMs were resolved by $12 \%$ sodium dodecyl sulfate polyacrylamide gel electrophoresis, electroblotted onto polyvinylidene difluoride membranes, and incubated with specific primary antibodies targeted to the protein of interest at the recommended dilutions. The blots were incubated with appropriate horseradish peroxidase-conjugated secondary antibodies. The semiquantitative detection of cross-reactive bands was performed by the ECL system. 


\section{Cellular Physiology Cell Physiol Biochem 2018;45:2421-2430

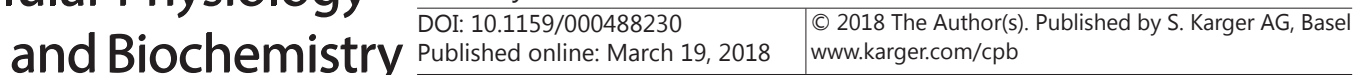

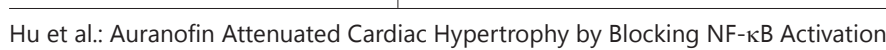

$N F-\kappa B$ activity assay

A nuclear extraction kit (KeyGEN, Nanjing, China) was used to extract the nuclear proteins from a section of the frozen tissues or CMs in strict accordance with the manufacturer's instructions. NF$\kappa B$ activity was assessed by an enzyme-linked immunosorbent assay specific for the NF- $\kappa B$ (p65) transcription factor (Cayman Chemicals, Ann Arbor, MI, USA) according to the manufacturer's protocol.

\section{Echocardiography}

At 9 weeks post-surgery, all treated and control group rats were weighed and anesthetized with chloral hydrate before clinical analysis. An IE33 echocardiographic system measured the transthoracic activity by two-dimensionally guided M-mode echocardiography (Philips Medical Systems, the Netherlands), which was equipped with a 15- $\mathrm{MHz}$ (s12) transducer. We adopted the following variables: the percentage of left ventricular (LV) posterior wall dimensions at diastole (LVPWd), LV internal dimensions at systole (LVIDs), LV fractional shortening (FS), and LV ejection fraction (EF).

\section{Statistical analysis}

Statistical analysis was performed using SPSS statistical software package version 17.0. Values were defined as the mean \pm standard deviation. Differences between group means were tested by oneway analysis of variance, followed by Tukey's post hoc test of significance. An alpha value of $\mathrm{P}<0.05$ was considered statistically significant.

\section{Results}

\section{Aur attenuated cardiac remodeling}

We determined whether the indices of cardiac remodeling could be attenuated by Aur administration commencing 1 week after AAC. At the end of 8 weeks of treatment, rats subjected to AAC displayed clear cardiac hypertrophy, which was apparent from the enhanced cardiac mass and increased heart weight/body weight (HW/BW), compared with the sham group. However, after administration of Aur for 8 weeks, the hypertrophic responses were diminished (Fig. 1AC). Moreover, hypertrophic markers including the expression of $\beta$-MHC were induced after AAC but markedly attenuated in the Aur-treated group (Fig. 1D).

\section{Impaired cardiac}

function after $A A C$ was improved by Aur

After 9 weeks postsurgery, we further determined whether Aur may attenuate AAC-induced left ventricular dysfunction using echocardiographic analysis (Fig. 2A). Nine weeks after AAC, the treated animals revealed markedly

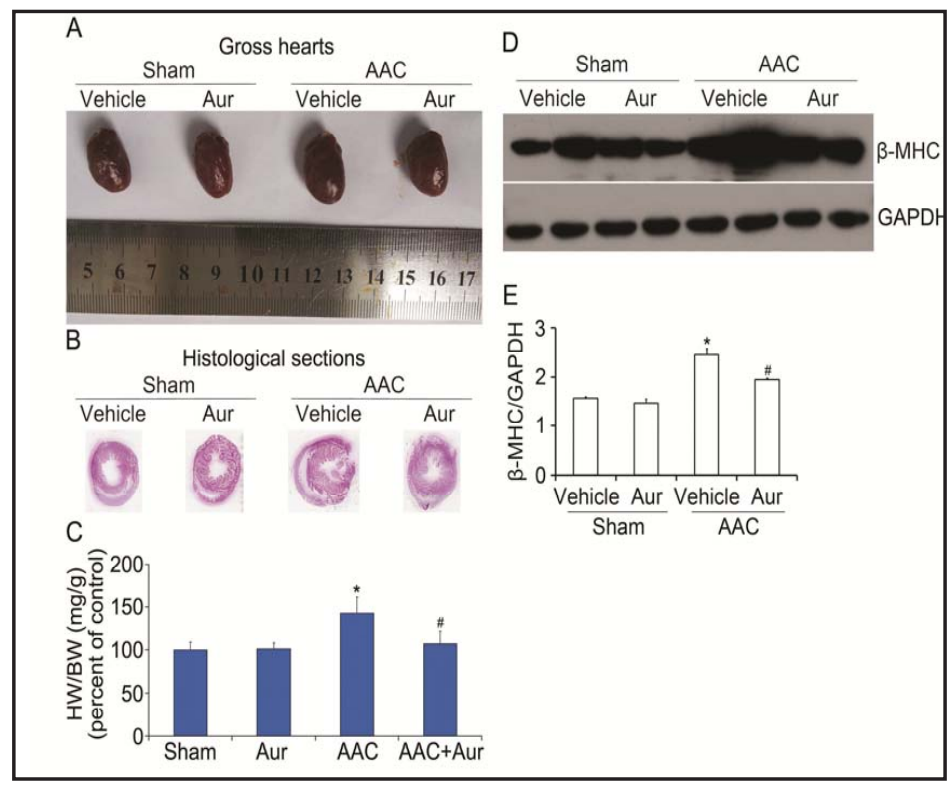

Fig. 1. Effects of auranofin (Aur) on cardiac hypertrophy. (A) Gross hearts. (B) Histological sections (hematoxylin-eosin staining). (C) Statistical analysis of the heart weight/body weight $(\mathrm{HW} / \mathrm{BW})$ ratio $(\mathrm{n}=$ 8). (D and E) The protein level of $\beta$-MHC was determined by western blot. GAPDH was used as an internal loading control. *, $\mathrm{P}<0.05$ vs. the corresponding sham group; ${ }^{\#}, \mathrm{P}<0.05$ vs. the $\mathrm{AAC}+$ vehicle group. 
enhanced chamber diameters and decreased EF and FS values. In addition, Aur notably improved LV function and attenuated cardiac dilation. Moreover, in AAC rats, LVPWd and LVID measurements were increased compared with sham rats at 9 weeks post-surgery, as shown by echocardiographic analysis; however, this effect was eliminated following Aur treatment (Fig. 2B). In addition, Aur-treated AAC rats exhibited better systolic functional parameters (i.e., EF and FS) compared with AAC rats (Fig. 2C).

Aur attenuated pressure overload-induced cardiac fibrosis

LV hypertrophy and subsequent heart failure are typically revealed by the appearance of fibrosis. Thus, we performed Masson's trichrome staining on serial sections of cardiac tissue to determine the progression of interstitial fibrosis in each group of treated rats. Rats subjected to AAC surgery showed marked interstitial fibrosis, which was attenuated by Aur treatment (Fig. 3A). Immunohis tochemistry assays were also used to determine the effects of Aur treatment on collagen type 1 expression in myocardial

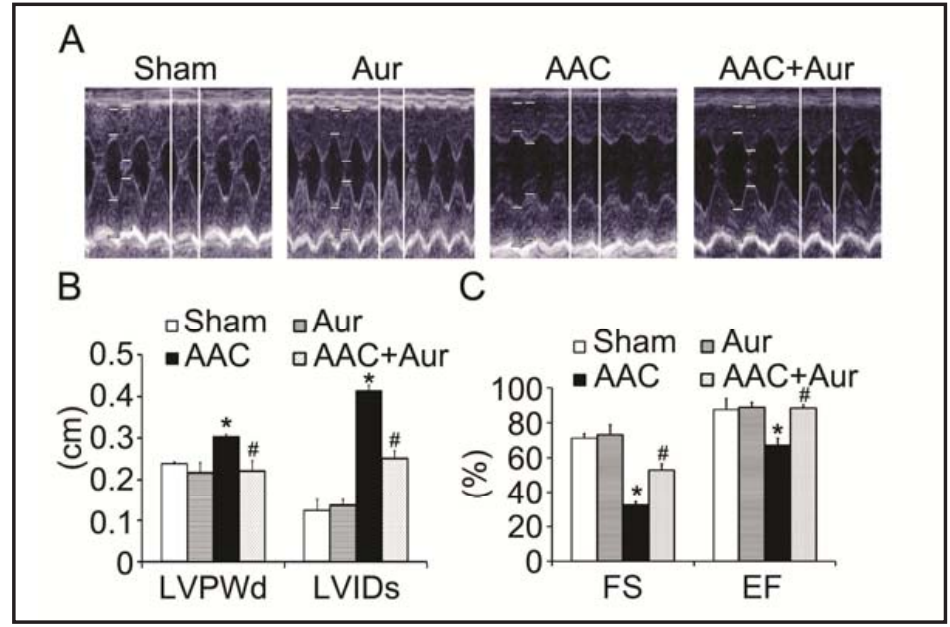

Fig. 2. Effects of auranofin (Aur) on echocardiography measurements. (A) Representative echocardiographic images. (B) Aur reduced the increased left ventricular (LV) diameters induced by AAC, including the LV posterior wall dimension at diastole (LVPWd) and LV internal dimensions at systole (LVIDs). (C) Aur attenuated AAC-induced changes in the ejection fraction (EF) and fractional shortening (FS). Data are presented as the mean \pm standard deviation $(\mathrm{n}=4)$. ${ }^{*}, \mathrm{P}<0.05$ vs. the corresponding sham group; ${ }^{\#}, \mathrm{P}<0.05$ vs. the $\mathrm{AAC}+$ vehicle group.

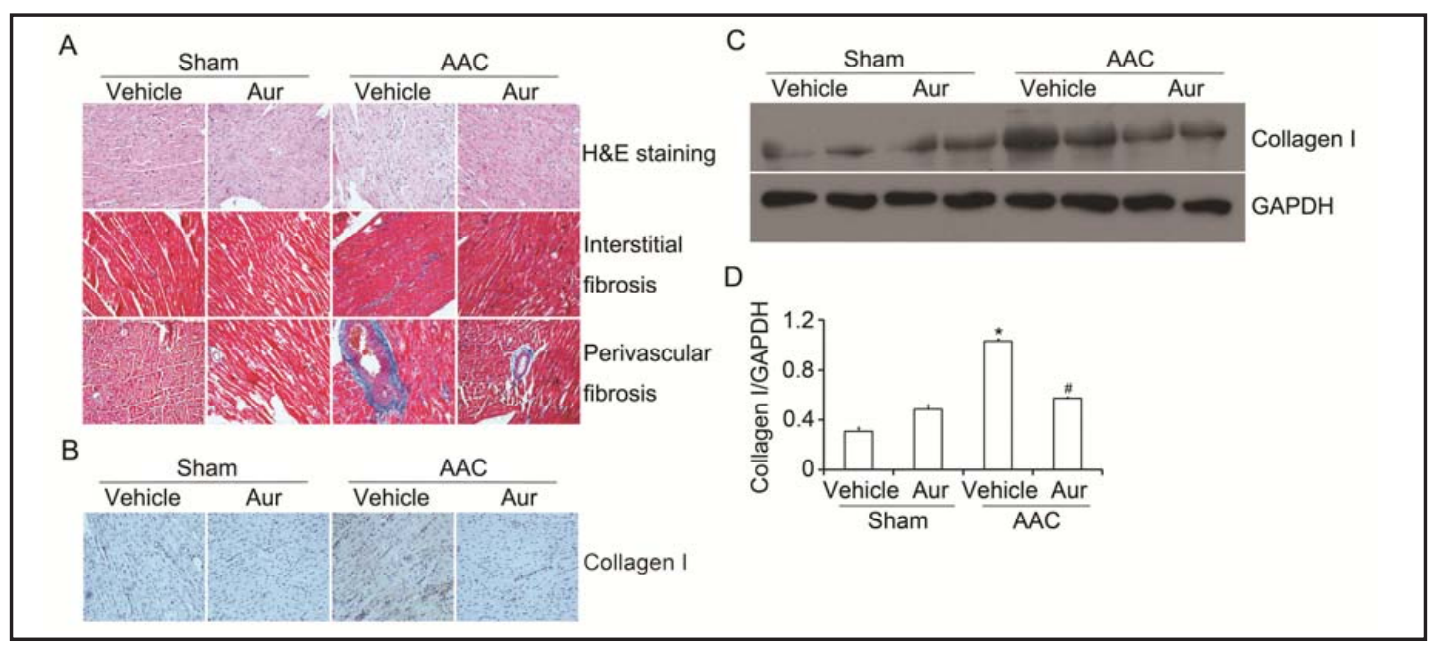

Fig. 3. Effect of auranofin (Aur) on cardiac fibrosis of the left ventricular (LV) myocardium. (A) Representative photomicrographs of Masson's trichrome staining of interstitial and perivascular fibrosis showing the myocardium in red and fibrosis in blue. (B) Collagen type I in the left ventricular myocardium was detected by immunochemistry $(200 \times)$. The immunostaining assays were performed using three independent left ventricular myocardial tissues. (C and D) The protein levels of collagen type I were assayed and detected by western blot. GAPDH was used as an internal loading control. *, $\mathrm{P}<0.05$ vs. the vehicle-treated sham group; \#, $\mathrm{P}<0.05$ vs. the vehicle-treated AAC group. 
Fig. 4. Effects of auranofin (Aur) on proteasome function and the activation of NF- $\mathrm{\kappa B}$. (A-D) Aur accumulated proteasome substrate proteins in the left ventricular myocardial tissues. Western blot was performed to detect the amount of ubiquitinated proteins (Ubs), K48- ubiquitinated proteins, and IкB $\alpha$. GAPDH was used as an internal loading control. *, $\mathrm{P}<0.05$ vs. the corresponding sham group; \#, $\mathrm{P}<0.05$ vs. the $\mathrm{AAC}+$ vehicle group. (E) Ubs, K48- ubiquitinated proteins, and IкB $\alpha$ in the left ventricular myocardium were also detected by immunochemistry (200x). The immunostaining assays were performed using three independent left ventricular myocardial tissues. (F) The activation of NF- $\kappa B$ was determined by enzyme-linked immunosorbent assay. ${ }^{*}, \mathrm{P}<0.01$

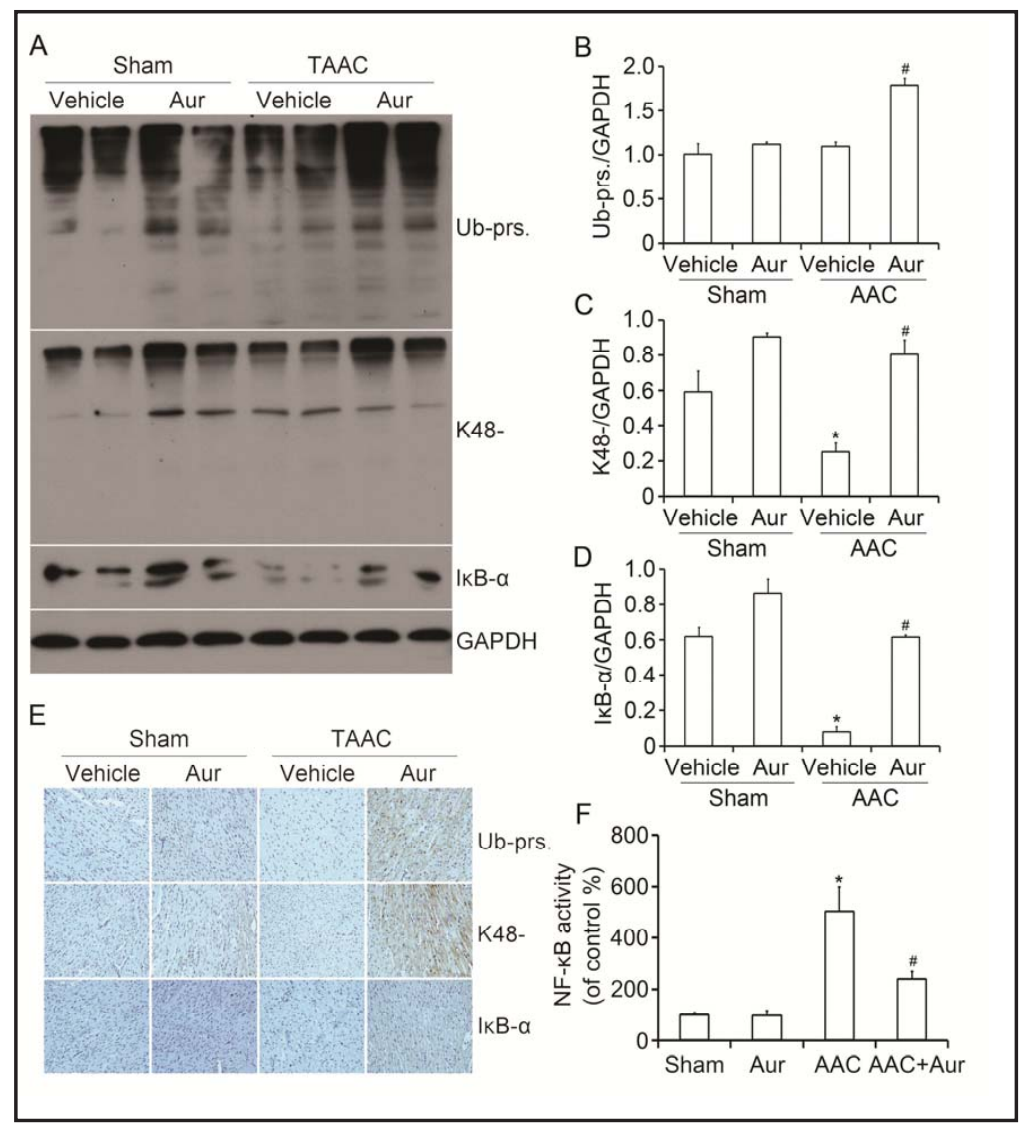
vs. the vehicle-treated sham group; ${ }^{*}, \mathrm{P}<0.01$ vs. the vehicle-treated AAC group.

tissue at 9 weeks post-surgery. High expression of collagen type-1 was noted in the hearts of AAC surgically-treated rats but attenuated by Aur treatment (Fig. 3B). Western blot analysis was used to evaluate the level of collagen type-1 protein in left ventricular tissue specimens, and the results resembled those of immunochemistry analysis (Fig. 3C-D).

\section{Aur treatment decreased proteasome activity and inactivation of the NF- $\kappa B$ signaling} pathway in cardiac tissue

Our previous studies showed that the 19S proteasome-associated DUB inhibitor, Aur, blocks the degradation of I $\mathrm{B} \alpha$ through a mechanism that requires proteasome inhibition [16]. Thus, we explored the ability of Aur to delay the expression of IкB $\alpha$ in myocardial cells following AAC surgery. Daily administration of Aur at $3 \mathrm{mg} / \mathrm{kg}$ induced an increase in I $\mathrm{B} \alpha$ accumulation at 9 weeks following AAC surgery (Fig. 4A). Moreover, Aur markedly enhanced the total amount of ubiquitinated proteins and K48-linked ubiquitinated proteins in the AAC-surgically treated group (Fig. 4A-D). Immunochemistry further demonstrated that the total amount of ubiquitinated proteins and K48-linked ubiquitinated proteins, and the expression of I $\mathrm{I} \mathrm{B} \alpha$ in the left ventricular myocardium were also increased in the Aur-treated AAC surgery group (Fig. 4E). Next, we attempted to unravel the mechanisms underlying the Aur-mediated protection of rat hearts. By measuring the NF- $\kappa B$ activity in cardiac tissues, we found that the levels were remarkably increased in the AAC group compared with the sham-treated group; however, the increased activity was quenched by treatment with Aur (Fig. 4F). These observations indicated that inhibition of the UPS by Aur might inactivate NF$\kappa \mathrm{B}$ signaling through a mechanism that is partly dependent on an increased accumulation of I $\mathrm{B} B \alpha$. 
Fig. 5. Effects of auranofin (Aur) on a neonatal rat primary myocardial cell hypertrophy model. (A) MTS assay was performed to assess cell viability after $24 \mathrm{~h}$ treatment with increasing concentrations of Aur or vehicle control., $\mathrm{P}<0.05$ vs. the vehicle-treated group; ${ }^{*}, \mathrm{P}<0.05$ vs. the Ang II/Aur-treated group. (B and $C$ ) The protein levels of $\beta$-MHC were assayed and detected by western blot. GAPDH was used as an internal loading control. *, $\mathrm{P}<0.05$ vs. the vehicle-treated group; \#, $\mathrm{P}<0.05$ vs. the Ang II-treated group. (D and E) Effects of Aur on collagen type I expression in cardiac fibroblasts with or without Ang II. The protein level of collagen type I was assayed and detected by western blot. GAPDH was used as an internal loading control. *, $\mathrm{P}<0.05$ vs. the vehicletreated group; \#, $\mathrm{P}<0.05$ vs. the Ang II-treated group.

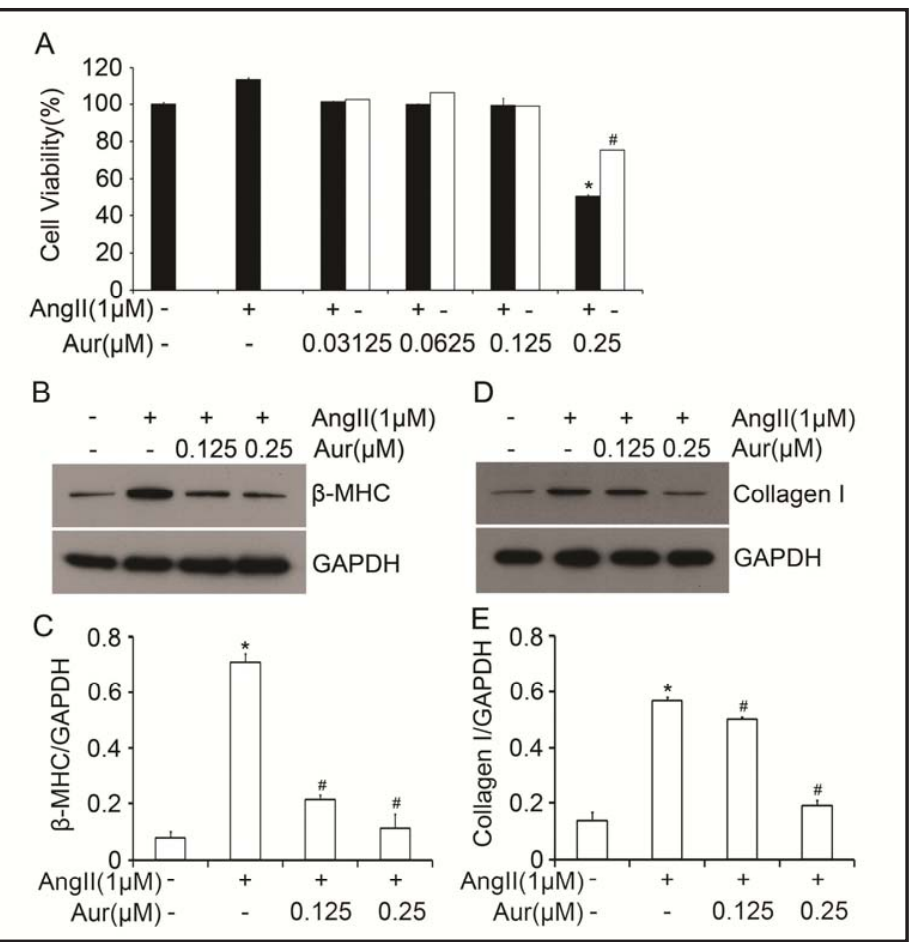

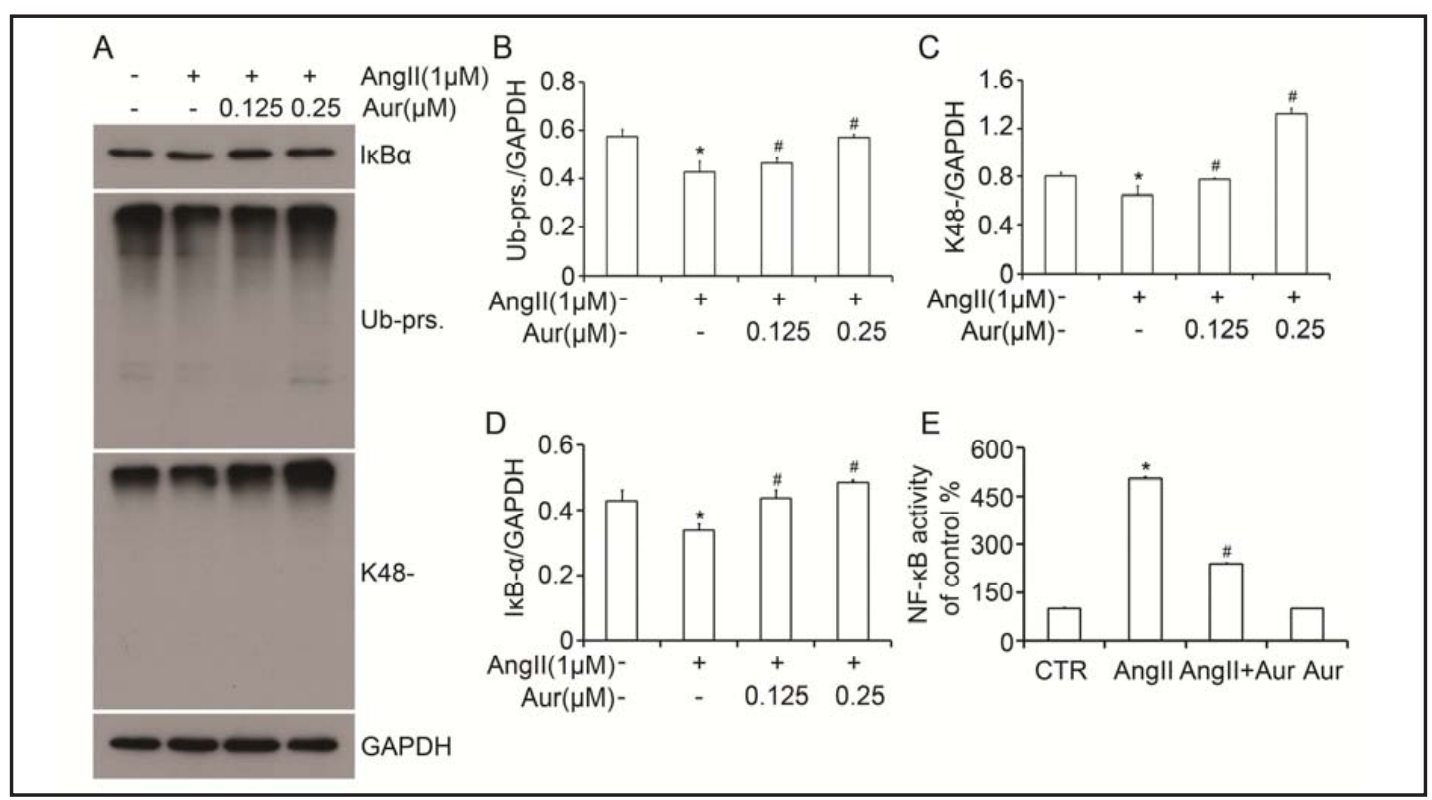

Fig. 6. Effects of auranofin (Aur) on proteasome function and the activation of NF- $\mathrm{BB}$ in myocardial cell hypertrophy induced by Ang II. (A-D) Aur accumulated proteasome substrate proteins in cardiomyocytes with or without Ang II. The protein levels of ubiquitinated proteins (Ubs), K48- ubiquitinated proteins, and IкB $\alpha$ were assayed and detected by western blot. GAPDH was used as an internal loading control. * $\mathrm{P}<0.05$ vs. the vehicle-treated group; \#, $\mathrm{P}<0.05$ vs. the Ang II-treated group. (E) Aur significantly reduced the NF- $\kappa \mathrm{B}$ activity induced by Ang II. *, P<0.05 vs. the vehicle-treated group; \#, $\mathrm{P}<0.05$ vs. the Ang II-treated group. 


\section{Cellular Physiology Cell Physiol Biochem 2018;45:2421-2430

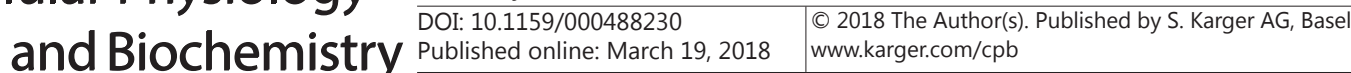 \\ Hu et al.: Auranofin Attenuated Cardiac Hypertrophy by Blocking NF-KB Activation}

Aur treatment inhibited CM hypertrophy and inactivated the NF- $\kappa B$ signaling pathway in CMs

The above results confirmed that Aur blocked the development of left ventricular hypertrophy induced by abdominal aortic constriction through inactivation of the NF- $\kappa B$ pathway. To further investigate this outcome, the results were verified in the neonatal rat primary myocardial cell hypertrophy model. We observed that Aur treatment did not exert cytotoxic effects on the CMs in the presence or absence of Ang II, although the highest dose of Aur $(0.25 \mu \mathrm{M})$ showed slightly cytotoxic effects (Fig. 5A). Thus, Aur concentrations of 0.125 $\mu \mathrm{M}$ and $0.25 \mu \mathrm{M}$ were used for the subsequent experiments. We found that Aur treatment markedly decreased the Ang II-induced expression of hypertrophic markers, $\beta$-MHC and collagen type-1 (Fig. 5B-E). In addition, Aur significantly enhanced the amount of total ubiquitinated proteins, K48-linked ubiquitinated proteins, and the expression of IкB $\alpha$ in CMs in the presence of Ang II (Fig. 6A-D). Aur treatment also decreased the activity of NF- $\kappa B$ in CMs in the presence of Ang II (Fig. 6E). Consistent with the in vivo data, the results found in the CMs also indicated that Aur inhibited cardiac hypertrophy by inactivation of the NF- $\mathrm{BB}$ pathway via inhibition of the UPS.

\section{Discussion}

Accumulating evidence indicates that the UPS is a key pathway that influences the onset of cardiac hypertrophy and subsequent heart failure. The UPS is a complex of ubiquitin, ubiquitin-activating enzymes (E1s), ubiquitin-conjugating enzymes (E2s), ubiquitin ligases (E3s), DUBs, and proteasomes. DUBs have been found to play a vital role in several intracellular processes including growth regulation, gene transcription, programmed cell death or apoptosis, and cell cycle progression via the regulation of three major checkpoints in the cycle [24]. DUBs are proven novel therapeutic targets for the treatment of tumors and other diseases. Currently, several DUB inhibitors are known to functionally affect only some specific DUBs. Thus, DUB inhibitors can treat several diseases with a high degree of target specificity. However, the capacity of these inhibitors to specifically treat cardiac hypertrophy is poorly understood. Previously, we reported that Aur is a 19S proteasome-associated DUB inhibitor [16]; thus, we examined its therapeutic effects on cardiac hypertrophy.

Nine weeks after AAC, the rats displayed cardiac hypertrophy marked by clinical endpoints such as increased HW/BW ratio and the expression of myocardial hypertrophy markers that trigger LV-associated dilation and dysfunction. Aur treatment almost completely attenuated the onset of cardiac hypertrophy, dysfunction, and fibrosis in rats following pressure overload. This phenomenon was associated with diminished functional activity of the proteasomes and that of the NF- $\kappa$ B signaling pathway.

Pressure overload can promote ventricular mechanical stress that initiates both cardiac hypertrophy and fibrosis $[18,25]$. A major intrinsic feature of fibrosis is increased cardiac collagen deposition [26-29]. In this study, we observed that Aur administration largely reduced the expression of collagen type I in pressure-overloaded hearts and cardiac fibroblasts induced by Ang II treatment, which suggested that blockade of cardiac fibroblast activation is a critical mechanism for Aur-mediated inhibition of the progression to cardiac fibrosis.

Furthermore, we determined the potential mechanisms underlying the capacity of Aur to decrease cardiac hypertrophy. The total amounts of ubiquitinated proteins, K48linked ubiquitinated proteins, and IкB $\alpha$ were decreased in the AAC surgical group and CM hypertrophy was induced by Ang II treatment. These results indicated that proteasomal activity had increased. Treatment with Aur induced the accumulation of total ubiquitinated proteins, K48-linked ubiquitinated proteins, and IкB $\alpha$ protein expression. We also found that the NF- $\kappa B$ activity increased significantly in the surgical AAC group and in CMs with hypertrophy induced by Ang II treatment. However, treatment with Aur suppressed the increased functional activity of NF- $\kappa B$.

Additionally, we determined that the capacity of Aur to slow cardiac remodeling might be mediated by reducing the activation of the NF- $\kappa B$ signaling pathway via partial blockade KARGER 


\section{Cellular Physiology Cell Physiol Biochem 2018;45:2421-2430

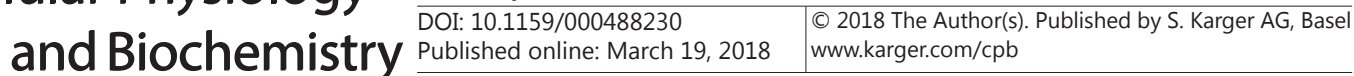

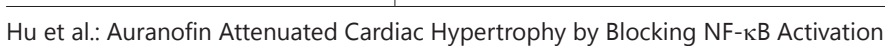

of proteasomal activity. Under normal conditions, cytoplasmic NF- $\kappa$ B is located in myocardial cells, and its inactive state depends on the combined functional activity of the inhibitor $\mathrm{I} \kappa \mathrm{B} \alpha$. Stimulation of myocardial cells, for example by hemodynamic changes, triggers the release of cytokines induced by activation of the IкB kinase (IKK) complex and subsequent phosphorylation of the I $\mathrm{KB} \alpha$ protein. This phosphorylated protein is ubiquitinated and degraded by the $26 \mathrm{~S}$ proteasome, which in turn provokes the release and nuclear translocation of NF- $\kappa \mathrm{B}$, which subsequently activates the transcription of specific target genes. Previously published studies have indicated that the NF- $\kappa B$ pathway is critical in the development of cardiac hypertrophy. Consequently, such studies indicated that inactivated NF- $\kappa \mathrm{B}$ signaling might stimulate the regression of cardiac hypertrophy induced by pressure overload [17, 18]. Thus, the ability of Aur to repress cardiac remodeling might inhibit IкB $\alpha$ degradation, which results in the inactivation of the NF- $\kappa B$ signaling pathway.

\section{Conclusion}

This study provided new evidence to suggest that the DUB inhibitor Aur interferes in the cardiac pathology resulting from pressure overload or Ang II. In addition, the underlying mechanisms include proteasome inhibition and NF- $\kappa B$ inactivation. However, previous studies have shown that Aur might have several cellular targets that collectively contribute to its biological effects through the inhibition of USP14 and UCHL5. Consequently, other underlying mechanisms that could be stimulated by Aur treatment for left ventricular hypertrophy need to be clarified in further studies. Nonetheless, the current data suggested that Aur has a remarkable therapeutic potential as an anti-hypertrophic agent in addition to its known biological activities as an anti-cancer drug.

\section{Acknowledgements}

The study was supported in part by the National Natural Science Foundation of China (81570259, 81400231, 81774100), the Natural Science Foundation of Guangdong Province (2017A030313796), the Science and Technology Planning Project of Guangdong Province (2014A020212330, 2016A020215167), the Education Features Innovative Project of Guangdong Province 2016KTSCX118, 2016KQNCX134), Pearl River S\&T Nova Program of Guangzhou (No. 201506010071), General Project from Guangzhou Education Commission (1201610098), Guangzhou Medical, Health Science, and Technology Project (20162A011018), and the Foundation of Guangzhou Medical University for Doctor Scientists (2016C17).

\section{Disclosure Statement}

The authors declare no conflicts of interest.

\section{References}

-1 Jessup M, Brozena S: Heart failure. N Engl J Med 2003;348:2007-2018.

2 Chen J, Normand SL, Wang Y, Krumholz HM: National and regional trends in heart failure hospitalization and mortality rates for medicare beneficiaries, 1998-2008. JAMA 2011;306:1669-1678.

-3 Hochstrasser M: Ubiquitin, proteasomes, and the regulation of intracellular protein degradation. Curr Opin Cell Biol 1995;7:215-223.

4 Nandi D, Tahiliani P, Kumar A, Chandu D: The ubiquitin-proteasome system. J Biosci 2006;31:137-155.

5 Depre C, Wang Q, Yan L, Hedhli N, Peter P, Chen L, Hong C, Hittinger L, Ghaleh B, Sadoshima J, Vatner DE, Vatner SF, Madura K: Activation of the cardiac proteasome during pressure overload promotes ventricular hypertrophy. Circulation 2006;114:1821-1828.

-6 Li N, Wang HX, Han QY, Li WJ, Zhang YL, Du J, Xia YL, Li HH: Activation of the cardiac proteasome promotes angiotension ii-induced hypertrophy by down-regulation of atrap. J Mol Cell Cardiol 2015;79:303-314.

7 Lee H, Park J, Kim EE, Yoo YS, Song EJ: Proteasome inhibitors attenuated cholesterol-induced cardiac hypertrophy in h9c2 cells. BMB Rep 2016;49:270-275. 


\section{Cellular Physiology Cell Physiol Biochem 2018;45:2421-2430 and Biochemistry Published \begin{tabular}{l|l} 
DOI: 1159/000488230 & $\begin{array}{l}\text { C) } 2018 \text { The Author(s). Published by S. Karger AG, Basel } \\
\text { www.karger.com/cpb }\end{array}$ \\
\hline
\end{tabular}}

Hu et al.: Auranofin Attenuated Cardiac Hypertrophy by Blocking NF-KB Activation

8 Ma Y, Chen Y, Yang Y, Chen B, Liu D, Xiong Z, Zhang C, Dong Y: Proteasome inhibition attenuates heart failure during the late stages of pressure overload through alterations in collagen expression. Biochem Pharmacol 2013;85:223-233.

-9iu S, Zhao C, Yang C, Li X, Huang H, Liu N, Li S, Wang X, Liu J: Gambogic acid suppresses pressure overload cardiac hypertrophy in rats. Am J Cardiovasc Dis 2013;3:227-238.

10 Zhao C, Liu S, Yang C, Li X, Huang H, Liu N, Li S, Wang X, Liu J: Gambogic acid moderates cardiac responses to chronic hypoxia likely by acting on the proteasome and nf-kappab pathway. Am J Cardiovasc Dis 2013;3:135-145.

11 D’Arcy P, Linder S: Proteasome deubiquitinases as novel targets for cancer therapy. Int J Biochem Cell Biol 2012;44:1729-1738.

12 D’Arcy P, Brnjic S, Olofsson MH, Fryknas M, Lindsten K, De Cesare M, Perego P, Sadeghi B, Hassan M, Larsson R, Linder S: Inhibition of proteasome deubiquitinating activity as a new cancer therapy. Nat Med 2011;17:1636-1640.

13 Madeira JM, Gibson DL, Kean WF, Klegeris A: The biological activity of auranofin: Implications for novel treatment of diseases. Inflammopharmacology 2012;20:297-306.

14 Mirabelli CK, Johnson RK, Sung CM, Faucette L, Muirhead K, Crooke ST: Evaluation of the in vivo antitumor activity and in vitro cytotoxic properties of auranofin, a coordinated gold compound, in murine tumor models. Cancer Res 1985;45:32-39.

15 Liu N, Huang H, Dou QP, Liu J: Inhibition of 19s proteasome-associated deubiquitinases by metal-containing compounds. Oncoscience 2015;2:457-466.

-16 Liu N, Li X, Huang H, Zhao C, Liao S, Yang C, Liu S, Song W, Lu X, Lan X, Chen X, Yi S, Xu L, Jiang L, Zhao C, Dong X, Zhou P, Li S, Wang S, Shi X, Dou PQ, Wang X, Liu J: Clinically used antirheumatic agent auranofin is a proteasomal deubiquitinase inhibitor and inhibits tumor growth. Oncotarget 2014;5:5453-5471.

17 Javan H, Szucsik AM, Li L, Schaaf CL, Salama ME, Selzman CH: Cardiomyocyte p65 nuclear factor-kappab is necessary for compensatory adaptation to pressure overload. Circ Heart Fail 2015;8:109-118.

18 Zhang Z, Qu X, Ni Y, Zhang K, Dong Z, Yan X, Qin J, Sun H, Ding Y, Zhao P, Gong K: Triptolide protects rat heart against pressure overload-induced cardiac fibrosis. Int J Cardiol 2013;168:2498-2505.

19 Chen B, Ma Y, Meng R, Xiong Z, Zhang C, Chen G, Zhang A, Dong Y: Mg132, a proteasome inhibitor, attenuates pressure-overload-induced cardiac hypertrophy in rats by modulation of mitogen-activated protein kinase signals. Acta Biochim Biophys Sin (Shanghai) 2010;42:253-258.

20 Liu N, Chai R, Liu B, Zhang Z, Zhang S, Zhang J, Liao Y, Cai J, Xia X, Li A, Liu J, Huang H, Liu S: Ubiquitinspecific protease 14 regulates cardiac hypertrophy progression by increasing gsk-3beta phosphorylation. Biochem Biophys Res Commun 2016;478:1236-1241.

-21 Liao Y, Liu N, Hua X, Cai J, Xia X, Wang X, Huang H, Liu J: Proteasome-associated deubiquitinase ubiquitinspecific protease 14 regulates prostate cancer proliferation by deubiquitinating and stabilizing androgen receptor. Cell Death Dis 2017;8:e2585.

22 Liu N, Huang H, Xu L, Hua X, Li X, Liu S, Yang C, Zhao C, Zhao C, Li S, Dou QP, Liu J: The combination of proteasome inhibitors bortezomib and gambogic acid triggers synergistic cytotoxicity in vitro but not in vivo. Toxicol Lett 2014;224:333-340.

23 Liu N, Kong T, Chen X, Hu H, Gu H, Liu S, Chen X, Yang Q Li A, Xiong X, Zhang Z: Ubiquitin-specific protease 14 regulates lps-induced inflammation by increasing erk1/2 phosphorylation and nf-kappab activation. Mol Cell Biochem 2017;431:87-96.

24 Reyes-Turcu FE, Ventii KH, Wilkinson KD: Regulation and cellular roles of ubiquitin-specific deubiquitinating enzymes. Annu Rev Biochem 2009;78:363-397.

25 Cao TT, Chen HH, Dong Z, Xu YW, Zhao P, Guo W, Wei HC, Zhang C, Lu R: Stachydrine protects against pressure overload-induced cardiac hypertrophy by suppressing autophagy. Cell Physiol Biochem 2017;42:103-114.

26 Chapman D, Weber KT, Eghbali M: Regulation of fibrillar collagen types i and iii and basement membrane type iv collagen gene expression in pressure overloaded rat myocardium. Circ Res 1990;67:787-794.

27 Lopez B, Gonzalez A, Varo N, Laviades C, Querejeta R, Diez J: Biochemical assessment of myocardial fibrosis in hypertensive heart disease. Hypertension 2001;38:1222-1226.

-28 Weber KT, Janicki JS, Shroff SG, Pick R, Chen RM, Bashey RI: Collagen remodeling of the pressureoverloaded, hypertrophied nonhuman primate myocardium. Circ Res 1988;62:757-765.

29 Liang J, Yin K, Cao X, Han Z, Huang Q, Zhang L, Ma W, Ding F, Bi C, Feng D, Pan Z, Liu Y: Attenuation of low ambient temperature-induced myocardial hypertrophy by atorvastatin via promoting bcl-2 expression. Cell Physiol Biochem 2017;41:286-295. 